\title{
AVALIAÇÃO DA EFICÁCIA DA IVERMECTINA SOBRE NEMATOIDES GASTRINTESTINAIS DE ASININOS Equus asinus Linnaeus, 1758
}

\section{EFFECTIVENESS EVALUATION OF IVERMECTIN ON GASTRINTESTINAL NEMATOIDS OF DONKEYS Equus asinus Linnaeus, 1758}

\section{EVALUACIÓN DE LA EFECTIVIDAD DE LA IVERMECTINA EN LOS NEMATOIDES GASTRINTESTINALES DE LOS BURROS Equus asinus Linnaeus, 1758}

Ruana Rafaela Lira Torquato Paiva ${ }^{1}$; Lígia Vanessa Leandro Gomes ${ }^{2}$; Maria de Lara Oliveira Lima $^{3}$; Eylha Pricilla Fernandes Menezes ${ }^{4}$; Josivania Soares Pereira ${ }^{5}$

DOI: https://doi.org/10.31692/978-65-991061-4-9.139-144

\section{INTRODUÇÃO}

Dentre os asininos, a espécie Equus asinus Linnaeus, 1978 apresenta características peculiares, que garantem sua grande utilização em regiões semiáridas, tais como: força, rusticidade e resistência, principalmente, ao clima quente e à escassez de alimentos (MARQUES et al., 2013). São usados para serviços de carga, transporte e, principalmente produção de híbridos (muares e bardotos), atividade esta que cresce a cada dia (SILVA et al., 2018).

Para utilização como modelos experimentais ou em asininoterapia, bem como evitar que os mesmos sejam disseminadores de patógenos a outros organismos (cavalos), estes animais precisam estar livres de uma carga parasitária alta e considerada patogênica. Infelizmente, é sabido que na busca por um controle total, no qual o objetivo é tratar com frequência suficiente para manter a carga parasitária e a contagem de ovos por grama de fezes (OPG) próximo de zero, cada vez mais se observa o uso indiscriminado e frequente dos antihelmínticos disponíveis, o que contribui para seleção de parasitos resistentes (FORTES, 2013). O objetivo do presente trabalho é avaliar a eficácia da Ivermectina sobre nematoides gastrintestinais de asininos Equus asinus Linnaeus, 1758.

\section{FUNDAMENTAÇÃO TEÓRICA}

É sabido que os equídeos silvestres ou domesticados podem ser parasitados por helmintos que afetam a sua saúde e bem-estar por ocasionar letargia, perda de peso, cólica

\footnotetext{
${ }^{1}$ Medicina Veterinária, Universidade Federal Rural do Semi-árido, ruanatorquato@ gmail.com

${ }^{2}$ Zootecnia, Universidade Federal Rural do Semi-árido, ligiavanessssa@ hotmail.com

${ }^{3}$ Medicina Veterinária, Universidade Federal Rural do Semi-árido, mlara.oliveira@ hotmail.com

${ }^{4}$ Medicina Veterinária, Universidade Federal Rural do Semi-árido, pryscylla_gt@ hotmail.com

${ }^{5}$ Docente adjunta da Universidade Federal Rural do Semi-árido, josigej@ufersa.edu.br
} 
potencialmente grave, diarreia e até a morte. (BEVILAQUA, 1988; CANEVER, 2012). Dentre os equídeos, os asininos, apesar de sua robustez, são animais vulneráveis às parasitoses. Nestes, a fauna parasitária é composta por mais de 100 espécies diferentes de helmintos gastrintestinais, incluindo Cestoda, Trematoda, Ascaridida e Strongylida (KRECEK, 2014; SOUSA, 2016). No semiárido brasileiro, entre os criadores, há uma tendência de considerar um conceito tradicional de que asininos são animais muito resistentes e que não requerem cuidados em seu manejo. Além disso, os estudos sobre doenças parasitárias em asininos têm sido negligenciados e, consequentemente, são raras as publicações científicas detalhadas sobre a biodiversidade parasitária assim como a sua prevalência, incidência e efeitos na saúde dos asininos (VILELA; FEITOSA, 2018). Isto representa uma grande preocupação, pois E. asinus são hospedeiros naturais de parasitas gastrintestinais do cavalo (Equus caballus).

Os protocolos terapêuticos são frequentemente administrados nos asininos com base na dosagem e nos intervalos recomendados para cavalos (GROSENBAUGH; REINEMEYER; FIGUEIREDO, 2011). Isso ocorre porque dados relativamente limitados estão disponíveis em parasitas internos e na farmacologia de drogas anti-helmínticas em jumentos e mulas quando comparados a ruminantes e cavalos, porque tais espécies foram consideradas de baixo interesse econômico e por isso negligenciadas em tais pesquisas. $\mathrm{O}$ tratamento anti-helmíntico é, portanto, um desafio, já que muitos fármacos não são licenciados para uso em asininos e muares (GOKBULUT; MCKELLAR, 2018). Contudo, jumentos e cavalos compartilham suscetibilidade à maioria dos endoparasitas e essas drogas têm se mostrado eficazes para o controle de infecção parasitária natural em jumentos mesmo em doses determinadas para cavalos (GROSENBAUGH; REINEMEYER; FIGUEIREDO, 2011).

\section{METODOLOGIA}

Foram utilizados vinte espécimes machos e fêmeas adultos de Equus asinus, provenientes de uma propriedade localizada no município de Felipe Guerra, Rio Grande do Norte. Coletou-se dos referidos asininos, amostras de fezes diretamente da ampola retal nos dias zero (antes do tratamento) e 14 dias após tratamento. Finalizadas as coletas, os mesmos permaneceram na sua propriedade de origem. Nos animais alocados no grupo controle (10 animais) apenas coletou-se as fezes enquanto que no grupo tratado (10 animais) foi aplicado uma única dose subcutânea de Ivermectina a $1 \%(0.2 \mathrm{mg} / \mathrm{kg}$ peso vivo) para posterior análise da resistência de endoparasitos. 
Para composição dos grupos experimentais, os animais tiveram sua massa corpórea medida e foram marcados através de colares confeccionados em plástico e com cores estabelecidas para cada grupo. Todos os procedimentos de manipulação e coleta de fezes nos animais foram executados conforme as recomendações propostas pela Comissão de Ética no uso de animais em pesquisa da UFERSA (projeto protocolo n. 23091.003873/2017-54, aprovado pela CEUA-UFERSA parecer número 07/2017).

Para realização da coleta das fezes, os animais foram contidos fisicamente (uso de cabresto) e em seguida foram submetidos a palpação retal. As amostras fecais coletadas diretamente da ampola retal foram enviadas ao Laboratório de Parasitologia Animal da UFERSA para análise. As fezes foram analisadas segundo técnica de Gordon e Whitlock (1939) modificado segundo Chagas et al. (2011). Para identificação dos gêneros e quando possível, espécies de endoparasitos foi realizado cultivo das fezes nos dias 0 e 14 dias póstratamento, segundo método de Roberts e O'Sullivan (1950) modificado (BLOOD et al., 1989; MEDICA; SUKHDEO, 1997).

Os dados obtidos foram expressos nos valores de média, desvio padrão bem como mínimos, máximos e porcentagem através do programa estatístico SPSS versão 23.0 Para distinguir a diferença estatística dos grupos experimentais, dentro e entre os momentos, foi utilizado o teste de Mann-Whitney. Para avaliar a eficácia do produto, foi usado o teste de redução na contagem de ovos por grama de fezes (TRCOF). Tomaram-se como dados, os resultados obtidos do exame de $\mathrm{OPG}$, que se fez as médias aritméticas do número de ovos nas fezes, para cada grupo tratado (OPGt), comparadas com as médias contadas no grupo controle (OPGc) determinada pela fórmula descrita por Coles et al., (1992).

\section{RESULTADOS E DISCUSSÃO}

Nas análises parasitológicas realizadas durante o período experimental e nos vinte animais estudados, foram encontrados ovos do tipo estrongilídeos, Strongyloides e larvas de Cyathostomum tipo A, Strongyloides westeri, Strongylus vulgaris e Posteriostomum sp.

Os animais do grupo tratado apresentaram no dia zero (D0) um OPG em média de 1435 ovos por gramas de fezes (valor mínimo 800 e máximo 3975 ovos por grama de fezes). Através da análise do teste de resistência realizado observou-se que os animais do grupo tratado apresentaram $100 \%$ de sensibilidade a ação da ivermectina a $1 \%$. Esse resultado foi percebido no décimo quarto dia (D14), no qual não houve detecção de ovos no OPG (Tabela $1)$.

No grupo controle, não houve diferença estatística $(p>0,05)$ nos diferentes momentos 
de avaliação, demonstrando que a infecção se manteve inalterada durante todo o estudo. Estes apresentaram em média um OPG de 1936 ovos por gramas de fezes (valor mínimo 800 e máximo 3700 ovos por grama de fezes) (Tabela 1).

Tabela 1 - Valores de média \pm desvio padrão de contagem de ovos por grama de fezes (OPG) nos asininos $(\mathrm{N}=10$ animais por grupo).

\begin{tabular}{|c|c|c|c|c|}
\hline \multirow{3}{*}{ OPG } & \multicolumn{4}{|c|}{ Momentos (dias) } \\
\hline & \multicolumn{2}{|c|}{ Grupo tratado } & \multicolumn{2}{|c|}{ Grupo controle } \\
\hline & D0 & $\mathrm{D}+14$ & D0 & $\mathrm{D}+14$ \\
\hline Média & $1435 \mathrm{~A}$ & OB & 1936A & $1477 \mathrm{~A}$ \\
\hline DP & 997 & 0 & 1133 & 1021 \\
\hline Máximo & 3975 & 0 & 3700 & 2650 \\
\hline Mínimo & 800 & 0 & 800 & 500 \\
\hline \% Redução & - & 100 & - & - \\
\hline
\end{tabular}

A, B. Médias seguidas de letras maiúsculas diferentes na linha significa diferença estatística ( $\mathrm{p}<0,05$ - Mann-Whitney) entre grupos nos diversos momentos.

D0. Primeiro dia de tratamento com ivermectina a $1 \%$.

D14. Décimo quarto dia após o tratamento com ivermectina a $1 \%$.

Fonte: Própria (2019)

Ao analisar a coprocultura do grupo tratado, considerando-se o dia zero (D0), os ovos cultivados desenvolveram-se em larvas de Cyathostomum tipo A (23\%), Strongyloides westeri (54\%) e de Strongylus vulgaris (23\%). No D14 percebeu-se a redução do potencial de larvas em 100\% nas coproculturas (Tabela 2).

No grupo controle a identificação das larvas infectantes obtidas das coproculturas, realizadas antes e após tratamento, possibilitou identificar larvas L3 com características morfológicas de Cyathostomum tipo A (44\%), de Strongyloides westeri (34\%) e de Strongylus vulgaris (22\%). Contudo, no D14 percebeu-se um aumento de Cyathostomum tipo A que passou aos 64\%, implicando na redução dos demais parasitos anteriormente encontrados, além da recuperação de 3\% de Posteriostomum sp. (Tabela 2).

Tabela 2 - Distribuição (\%) dos helmintos encontrados nas coproculturas realizadas antes (dia zero) e após o tratamento (14 dias) nos asininos ( $\mathrm{N}=10$ animais por grupo).

\begin{tabular}{ccccccc}
\hline \multirow{2}{*}{ Helmintos } & \multicolumn{3}{c}{ Dia 0} & & \multicolumn{3}{c}{ Dia 14 } \\
\cline { 2 - 3 } \cline { 6 - 7 } \cline { 5 - 7 } Controle $\%$ & Tratado $\%$ & & Controle \% & Tratado \% \\
\hline Cyathostomum Tipo A & 44 & 23 & & 64 & 0 \\
Strongyloides westeri & 34 & 54 & & 23 & 0 \\
Strongylus vulgaris & 22 & 23 & & 10 & 0 \\
Posteriostomum sp. & 0 & 0 & & 3 & 0 \\
\hline Total & 100 & 100 & & 100 & 100 \\
\hline
\end{tabular}

Fonte: Própria (2019)

Os resultados obtidos neste trabalho sugerem que os animais estavam com uma elevada infecção por formas adultas de estrongilídeos e Strongyloides sp. Considerando que uma contagem de 800 ovos por gramas de fezes já é um indicativo de infecção com 
necessidade de tratamento anti-helmíntico, o grau de parasitismo dos animais foi, portanto, considerado adequado para a avaliação do presente estudo (CHAGAS et al, 2011). A recuperação de ovos do tipo estrongilídeos em asininos já foi descrita de forma cosmopolita por muitos autores (FERREIRA et al., 2014; ASOKAN et al., 2016; ANTENEH; GETACHEW, 2017 ATTIA; KHALIFA; ATWA, 2018). Da mesma forma é descrito para ovos do tipo Strongyloides (WELLS et al., 1998; LUCENA; FIGHERA; BARROS, 2012).

No entanto, os valores mínimos e máximos de recuperação desses ovos divergem de Carvalho et al. (2007), que em Portugal, avaliando o grau de parasitismo em asininos estabulados, encontraram níveis de OPG de estrongilídeos, que variaram entre 200 e 2.000 ovos por grama de fezes. No presente caso, entre os dois grupos avaliados, os valores mínimos e máximos ficaram 800 e 3975 ovos por grama de fezes, respectivamente. Aproximando-se mais dos achados de Ferreira et al. (2014) que obtiveram valores de 600 a 6.900 OPG.

As larvas de terceiro estádio de Cyathostomum foram relativamente predominantes em ambos os grupos, confirmando a importância crescente dos estrongilídeos da subfamília Cyathostominae nos asininos do Brasil. Segundo Pfister e van Door (2018), a maioria dos estudos classificam os ciatostomíneos como uma entidade única, embora esse grupo englobe muitos morfotipos. Os valores médios do OPG do grupo tratado nos dias D0 e D14 foram significativamente menores $(\mathrm{p}<0,05)$ que os do grupo controle (Tabela 1). Portanto, os resultados do presente estudo com asininos naturalmente infectados demonstraram a eficácia da ivermectina, avaliada pelo teste de redução da contagem de ovos nas fezes, conforme relatado por outros autores (MADEIRA DE CARVALHO et al, 2007; DEDAR et al., 2011).

\section{CONCLUSÕES}

Nas análises parasitológicas realizadas durante o período experimental e nos vinte animais estudados, foram encontrados ovos do tipo estrongilídeos e Strongyloides identificouse larvas do tipo Cyathostomum tipo A, Strongyloides westeri, Strongylus vulgaris e Posteriostomum sp.

Os resultados do presente estudo com asininos naturalmente infectados demonstraram a eficácia de $100 \%$ da ivermectina sobre os nematoides gastrintestinais de Equus asinus.

\section{REFERENCIAS}

ASOKAN, S. et al. Study of endoparasitic infection in donkeys - a report. International [143] 
Journal Of Science, Environment Issn 2278-3687 (o) And Technology, v. 5, n. 6, p. 45454549, 2016.

ATTIA, M. M.; KHALIFA, M. M.; ATWA, M. Th..The prevalence and intensity of external and internal parasites in working donkeys (Equus asinus) in Egypt. Veterinary World, v. 11, n. 9, p. 1298-1306, 2018.

CANEVER, R.J. Diagnóstico da resistência anti-helmíntica em cíatostomineos de equinos por meio de testes in vivo e in vitro. 2012. 99p. Dissertação (Mestrado em Ciências Veterinárias) - Universidade Federal do Paraná, Curitiba, 2012.

CHAGAS, A. C. S.; NICIURA, S. C. M.; MOLENTO, M. B. Manual Prático: metodologias de diagnóstico da resistência e de detecção de substâncias ativas em parasitas ruminantes. Brasília: Embrapa Informação Tecnológica, 2011, 153 p.

DEDAR, R. K. et al. Therapeutic evaluation of ivermectin against Endoparasites of donkey. Veterinary Practitioner, v. 12, n. 1, p. 86-87, 2011.

FERREIRA, G. M. S. et al. Parasitismo gastrintestinal e hematologia em equinos e asininos da mesorregião da aglomeração urbana, São Luiz, Maranhão. Archives Of Veterinary Science, v. 19, n. 2, p. 22-30, 2014.

FORTES, F. S.; MOLENTO, M. B. Resistência anti-helmíntica em nematoides gastrintestinais de pequenos ruminantes: avanços e limitações para seu diagnóstico. Pesquisa Veterinária Brasileira, v. 33, n. 12, p. 1391-1402, 2013.

GOKBULUT, C.; MCKELLAR, Q. A.. Anthelmintic drugs used in equine species. Veterinary Parasitology, v. 261, p. 27-52, 2018.

GROSENBAUGH, D. A.; REINEMEYER, C. R.; FIGUEIREDO, M. D.. Pharmacology and therapeutics in donkeys. Equine Veterinary Education, v. 23, n. 10, p. 523-530, 2011.

LUCENA, R. B.; FIGHERA, R. A.; BARROS, C. S.L.. Mortalidade em potros associada ao parasitismo por Strongyloides westeri. Pesquisa Veterinária Brasileira, v. 32, n. 5, p. 401404, 2012.

MARQUES, D.D.; NÓBREGA NETO, P.I.; CARVAL, K. S. Emprego da cola de cianoacrilato em feridas cutâneas de asininos. Ciência Animal Brasileira, v. 14, n. 1, p. 7480, 2013.

MEDICA, D.L.; SUKHDEO, M.V.K. Role of lipids in the transmission of the infective stage (L3) of Strongylus vulgaris (Namatoda: Strongyloidea). Journal of Parasitology, v. 83, n. 5, p. 775-779, 1997.

SILVA, P. C. A. et al. Taxa de recuperação e características morfológicas de embriões muares (Equus caballus $x$ Equus asinus). Pesquisa Veterinária Brasileira, v. 38, n. 7, p. 1453-1457, 2018. 